\title{
Constructing A Novel Signature Based on Immune-Related Lncrna to Improve Prognosis Prediction of Cervical Squamous Cell Carcinoma Patients
}

\author{
Dongchun Qin ( $\nabla$ qindongchun@zzu.edu.cn ) \\ First Affiliated Hospital of Zhengzhou University \\ Xuefeng Lv \\ First Affiliated Hospital of Zhengzhou University \\ Lu Liu \\ First Affiliated Hospital of Zhengzhou University \\ Pengxiang Li \\ First Affiliated Hospital of Zhengzhou University \\ Yingying Yuan \\ Henan Chest Hospital \\ Mengle Peng \\ The Third People's Hospital of Henan Province \\ Huifang Jin \\ First Affiliated Hospital of Zhengzhou University
}

\section{Research Article}

Keywords: Cervical squamous cell carcinoma, LncRNA, Prognosis, Immune infiltration, Immune checkpoint proteins, Chemotherapeutics

Posted Date: July 19th, 2021

DOI: https://doi.org/10.21203/rs.3.rs-640056/v1

License: (ㅇ) (i) This work is licensed under a Creative Commons Attribution 4.0 International License. Read Full License 


\section{Abstract}

We downloaded gene expression data, clinical data, and somatic mutation data of cervical squamous cell carcinoma (CSCC) patients from The Cancer Genome Atlas (TCGA) and Genotype-Tissue Expression (GTEx) databases. Predictive IncRNAs were screened using univariate analysis and lasso regression, and risk score of each patient were calculated according to the expression levels of IncRNAs and regression coefficients to establish a risk model that could be a novel signature. We assessed the correlation between immune infiltration status, chemotherapeutics sensitivity, immune checkpoint proteins (ICP) and the signature. Therefore, we selected 11 immune-related IncRNAs (WWC2,AS2, STXBP5.AS1, ERICH6.AS1, USP30.AS1, LINC02073, RBAKDN, IL21R.AS1, LINC02078, DLEU1, LINC00426, BOLA3.AS1) to construct the risk model. Patients with high risk had a shorter survival time than those with low risk. Risk scores in the signature were negatively correlated with macrophage M1, macrophage M2, and T cell CD8+. The expression levels of ICP such as PD-1 were substantially higher in the low-risk group. For chemotherapeutic agents, high-risk scores were associated with higher half-inhibitory concentrations (IC50) of cisplatin. These findings suggested that the risk model can be a novel signature for predicting CSCC patients' prognosis, and it also can be used to formulate clinical treatment plans for CSCC patients.

\section{Introduction}

Cervical squamous cell carcinoma is the fourth dominant cause of cancer-related deaths in women worldwide, accounting for 80$85 \%$ of all cervical cancer diagnoses.[1, 2]. With human papillomavirus (HPV) vaccination and early-stage screening, the incidence rate of CSCC has decreased significantly. Surgery, radiotherapy, and chemotherapy have been commonly used for cervical cancer patients, but the 5-year survival rate is still not satisfactory because of advanced stage, relapse, metastasis, and drug resistance[3-5]. Although the use of immunotherapy checkpoint inhibitors has been successful in the treatment of various cancers[6], the development and application of immunotherapy for CSCC patients are restricted because the positive response remains low[7]. Thus it is crucial to provide CSCC with new therapeutic targets and to find novel biomarkers for its early diagnosis and prognosis.

Long non-coding RNAs (IncRNAs) are a class of non-coding transcripts with sequences longer than 200 nucleotides that can mediate post-transcriptional modifications. [8, 9]. They play a key role in immune activation, cancer immunity, antigen presentation, and immune cell infltration[10,11]. For example, IncRNA CamK-A was found to be highly expressed in various human cancers and could regulate $\mathrm{Ca}^{2+}$-signaling-mediated tumor microenvironment remodeling[12]. Also, in colorectal cancer cells, overexpression of HLA-FAS1 could repress miR-375 and promote the expression of PFN1, further parting macrophages toward M2 phenotype and exacerbating tumorigenesis[13]. EGFR promoted immune escape of hepatocellular carcinoma cells and Treg cell differentiation through specific binding to EGFR protein [14]. In addition to participating in the tumor microenvironment, IncRNAs can also affect the response to immunotherapy[15], which is rarely reported in CSCC patients.

In this study, we used the RNA sequencing data of CSCC patients in the Cancer Genome Atlas (TCGA) and Genotype-Tissue Expression (GTEx) database to identify irlncRNA, and established an 11-irlncRNA signature based on a LASSO Cox regression analysis. Subsequently, we explored the company between the signature of 11-irlncRNA and several disease characteristics in CSCC patients. Drug sensitivity analysis also was performed to improve drug therapy. Collectively, the present study may provide a strategy to predict prognosis and immune-related therapeutic targets in CSCC patients.

\section{Results}

\section{Flow Chart}

We showed the general flow of the study in Fig. 1.

\section{Construction and validation of the Risk Assessment Model.}

Data analysis of TCGA and GTEx databases produced 1,081 differentially expressed IncRNAs (DElncRNAs), of which 224 were DEirlncRNAs (Figure. 2a) and 11 of those were related to prognosis in CSCC samples. These 11 DEirlncRNAs were subjected to univariate analysis(Figure. 2b) and LASSO (least absolute shrinkage and selection operator) regression analysis (Figure. 2c,d), and a risk signature including these 11 DEirlncRNAs was constructed. 11 DEirlncRNAs were dominated by moderate or weak correlations, suggesting that they might play a role in similar biological processes (Figure. 2e). The risk scores for each patient was computed using 
Risk scores $=$ WWC2.AS2*0.223 + STXBP5.AS1*0.274 + ERICH6.AS1 $*-0.182+$ USP30.AS1 $*(-0.055)+$ LINC02073* $(-0.043)+$

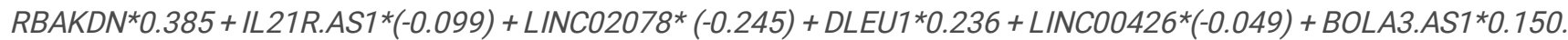

The AUC-ROC of the risk model for OS (overall survial) was $0.801,0.846$, and 0.847 at 1,3 and 5 years(Figure.3a), respectively. Using the AIC(Akaike Information Criterion) value (Fig. 3b), selected the maximum inflection point as the demarcation cut-off point of the 5year ROC (Receiver operating characteristics) curve. Using this cut-off point, we obtained a high-risk group of 115 patients and a lowrisk group of 189 patients. As shown by Kaplan-Meier analysis, patients in the high-risk group lived for a shorter period of time than those in the low-risk group (Fig. 3c). Figure 3d depicted the distribution of risk levels between the low-risk group and the high-risk group. Figure 3e showed the survival time of patients and the survival status in two risk groups, and Figure.3f displayed the corresponding expression standards of the 11 irlncRNA for each patient.

\section{PCA further verifies the grouping ability of the risk model}

Based on the expression profiles of 11 irlncRNAs in the risk model, 224 irLncRNA, immune genes and whole genes, we used Principal component analysis (PCA)to research the distribution patterns of the high-risk and low-risk groups in the CSCC patients (Figure. 4a-d). While based on the 11 irLncRNA, these two groups were significantly different, indicating that the prognostic signature can distinguish two groups well.

\section{Clinical evaluation by the risk model}

We investigated the relationship among risk signature, clinicopathological characteristics, and $11 \mathrm{DEirlncRNAs.} \mathrm{T} \mathrm{stage,} \mathrm{N}$ stage, and just were related to the risk group (Figure. 5a). Further, patients at T4 had higher risk scores than at T1, whereas patients at Stage IV had higher risk scores than at Stage III (Figure. 5b).

\section{Immune-related pathways of the two risk groups}

The results of Gene Set Enrichment Analysis (GSEA) (Table 1) suggested that three of the TOP 5 pathways enriched in the low-risk group were related to immunity, including autoimmune thyroiditis, primary immunodeficiency, and intestinal immune network (Figure. $6)$.

Table 1

Top10 pathway enriched in the low-risk group

\begin{tabular}{|c|c|c|c|c|}
\hline NAME & ES & NES & NOM p-val & FDR q-val \\
\hline KEGG_AUTOIMMUNE_THYROID_DISEASE & -0.78236496 & -2.1533732 & 0 & 0 \\
\hline KEGG_PRIMARY_IMMUNODEFICIENCY & -0.82993656 & -2.0816772 & 0 & 3.00E-04 \\
\hline KEGG_CELL_ADHESION_MOLECULES_CAMS & -0.6021577 & -2.0348785 & 0 & 0.001877939 \\
\hline KEGG_INTESTINAL_IMMUNE_NETWORK_FOR_IGA_PRODUCTION & -0.75884634 & -2.038879 & 0 & 0.001890281 \\
\hline KEGG_ALLOGRAFT_REJECTION & -0.8792095 & -2.0443735 & 0 & 0.002214069 \\
\hline KEGG_TYPE_I_DIABETES_MELLITUS & -0.78216136 & -2.002412 & 0 & 0.003311558 \\
\hline KEGG_ASTHMA & -0.76662517 & -1.9769996 & 0.001908397 & 0.005337439 \\
\hline KEGG_HEMATOPOIETIC_CELL_LINEAGE & -0.6412886 & -1.9576701 & 0.002024292 & 0.005633736 \\
\hline KEGG_VIRAL_MYOCARDITIS & -0.63541704 & -1.9355513 & 0.002024292 & 0.006129693 \\
\hline
\end{tabular}

\section{Immune landscape in CSCC patients}

The risk scores were negatively associated with macrophages $M 1$, macrophages $M 2$, myeloid dendritic cell, CD8 + T cells, whereas it was positively associated with macrophages MO (Figure. 7a). Meanwhile, the difference analysis also showed, CD $8+T$ cells were significantly higher in the group of low-risk, and macrophages $\mathrm{M} 0$ significantly higher in the group of high-risk (Figure. 7c). The proportion and infiltrating level of immune cells in 309 CSCC samples is shown in Figure. 7(b), and CD4 + T cells made up the highest 
percentage (Figure. 7d). There was a weak or moderate correlation between 22 tumor immune cells (Figure. 7e). Six LncRNAs among the risk signature had better relationship with immune cells (|correlation coefficients $\mid>0.4)$ (Figure. $7 f)$.

Six immune checkpoint proteins PD-L1, PD-1, CTLA-4, LAG-3, TIGIT and TIM-3 were expressed highly in the low-risk group (Figure.7g), suggesting that the immunosuppressive microenvironment led to a poor prognosis for low-risk patients. The prediction results of the tumor immune dysfunction and exclusion (TIDE) algorithm showed that low-risk patients score higher than high-risk patients, which indicated that low-risk patients responded better to immunotherapy. (Figure.7h).

\section{The significance of the model in clinical treatment}

CSCC tumors were classified into two clusters containing 64 and 242 members. (Figure. 8a). The two subtypes identified by the Nonnegative matrix factorization (NMF) algorithm were confirmed by Principal component analysis (PCA) (Figure. 8b). Cluster1 mainly contained patients with low-risk scores, while the high-risk and low-risk patients in cluster2 were basically the same (Figure. 8c). PDL1, PD-1, CTLA-4, LAG-3, TIGIT and TIM-3 in cluster 1 had significantly higher expression than in cluster 2 (Figure. 8d). Expression of immune cells like CD8 + T cells, macrophages M1, T cells follicular helper, T cells regulatory (Tregs) and Mast cells activated expressed was higher in cluster 2, whereas that of macrophages M0 was higher in cluster 1 (Figure. 8e).

\section{The significance of the model in clinical treatment}

Our study demonstrated that the high-risk group had a higher half-maximal inhibitory concentration(IC50) of cisplatin $(p<0.05)$ (Figure. 9), this suggested that the low-risk patients might be better suited for chemotherapy.

\section{Construction and assessment of predictive nomograms}

A nomogram was constructed to predict the 1-, 3- and 5-year OS incidences (Figure. 10a). Calibration curves and ROC analyses showed good predictive performance of the nomogram in predicting patient survival (Figure. 10b,c). The Decision Curve Analysis (DCA) showed that the nomogram has good clinical efficacy (Figure. 10d,e), suggesting that it can predict the survival of CSCC patients.

\section{Discussion}

It has been suggested that IncRNAs play a key role in the adjustment of the immune system and the tumor microenvironment[10]. Immune-related IncRNAs are prognostic markers of various types of cancer[16] linked to immune cell infiltration and are a potential target for cancer treatment[17]. IncRNA SNHG14 has been shown to be involved in the activation of the JAK-STAT pathway in cervical tumor cells [18]. In cervical cancer HeLa cells, the STAT3 binding sequence in the enhanced region of IncRNA MALAT1 proved to be the key to the activation of the MALAT1 promoter induced by IL-6 or STAT3 [19]. In the eleven immune-related IncRNAs of the risk model, STXBP5-AS1 targets miR-96-5p/PTEN axis to drive cervical cancer cell proliferation and invasion[20], and DLEU1 promotes cervical cancer cell proliferation and invasion via the miR-381/HOXA13 axis[21].

We used the AIC value to get the best cutoff point for the model fitting. The prognostic model performed better in distinguishing between risk groups of high and low. Patients with low-risk showed a favorable prognosis, which suggested that our model may be able to stratify risk. GSEA revealed that immune-related pathways were significantly enriched in the low-risk group. We also analyzed the tumor immune infiltration and efficacy of chemotherapy drugs in the treatment of CSCC, which determined that this model worked well. In this study, consensus clustering based on NMF was used to define CSCC subtypes. The CSCC sample could be separated into two subtypes with distinct molecular profiles with differences in immune cell infiltration and checkpoint-related biomarkers. A nomogram was established to show the exact agreement between the observation rate and the predicted rate of 1-year, 3-year, and 5year OS. The nomogram had better prediction accuracy and higher clinical efficacy. passed calibration curve and DCA verification.

The ability of immunotherapy depends on the immunogenicity of the tumor microenvironment, so the understanding of the tumor microenvironment is the key to assessing the possibility of immunotherapy[22]. Immune cell infiltration can be a predictive biomarker of cancer immunotherapy [23]. Patients with more CD $8+T$ cell infiltration get a better response from pembrolizumab than those with less infiltration [24]. Macrophages suppressed T cell recruitment and regulated other aspects of tumor immunity, they can be regulators of tumor immunity and immunotherapy[25]. We used seven accepted methods to estimate the correlation between risk scores and immune cells, the methods include XCELL[26], QUANTISEQ[27], MCPcounter[28], EPIC[29], TIMER[30, 31], CIBERSORT[32], and CIBERSORT-ABS[33]. By integrating analysis, our results revealed that the risk model was negatively associated with 
macrophages M1, macrophages M2, and CD8 + T cells, meanwhile CD8 + T cells were significantly higher in the low-risk group. We can assume that patients in the low-risk group may be more suitable for immunotherapy.

Cancer cells activate inhibitory immune checkpoint pathways to prevent the occurrence of autoimmunity[34], therefore, suppression of the immune checkpoint approach is a promising treatment. Evidence suggests that immunotherapy is a novel therapeutic strategy for the treatment of cervical cancer[35]. Anti-CTLA-4, anti-PD-1, and anti-PD-L1 therapies are possible treatment options for cervical cancer, studies show[36-38]. Furthermore, in a well-defined clinical study of 115 cervical cancer patients, PD-L1 expressed in $19 \%$ of cervical tumors, and more than $50 \%$ of tumor-infiltrating CD8 + T cells expressed PD-1[39]. We explored the relationship between several common immune checkpoint proteins in the treatment of cervical cancer and risk models, showing that all six immune checkpoints were higher in the group of low-risk. Thus under this model, it suggested that patients with low-risk may benefit more from immunotherapy. The predictions of the TIDE algorithm indicated that patients with low-risk subtypes had a better response to immunotherapy, which was consistent with the above-mentioned studies. Our risk model also showed that low-risk patients were more sensitive to cisplatin, a small-molecule platinum compound that treat recurrent cervical cancer effectively[40], so we can formulate chemotherapy regimens for low-risk patients.

In our risk model, the risk scores are related to the clinical stage, and the risk scores of early cervical cancer are lower than that of the advanced stage. From the joint analysis above, We can assume that early-stage patients may be better suited for immunotherapy and appropriate chemotherapy.

\section{Materials And Methods}

\section{Processing data and obtaining immune-related IncRNAs}

The gene expression data, clinical data, and somatic mutation data of CSCC patients were acquired from the TCGA index. The gene expression profiles of 10 normal cervical tissues were obtained from the GTEx database. The GTF file from Ensembl was used to annotate and distinguish mRNAs and IncRNAs. GSEA was applied to screen immune-related pathways; genes in these pathways were identified as immune genes. We used R software packages "limma" and "edgeR" to filter DElncRNAs, with |log2FC|>2 and FDR q-value $<0.05$. DElncRNAs with immune gene correlation coefficients greater than 0.5 and $p$-value $<0.001$ were selected as irlncRNAs

\section{Construction of risk model}

Univariate cox analysis was used and also LASSO[41] were carried out to screen for markers. The clinical features were chosen based on the best penalty parameter $\lambda$. We used the coefficients obtained from LASSO to calculate the risk scores.

$$
\text { riskScore }=\sum_{i=1}^{n} \text { Coef } * x_{i} \text {, }
$$

where Coefi is the coefficients and $x i$ is the fragments per-kilobase million(FPKM) value of each irlncRNA. We can calculate the risk scores of each patient according to this equation.

\section{Validation of the risk model}

The ROC for 1, 3, and 5 years of the model were calculated. The Akaike Information Criterion (AIC) value of the ROC curve was evaluated to select the maximum inflection point, which was considered to be the key point for distinguishing high-risk and low-risk groups. We used Kaplan-Meier analysis to explore the prognostic value based on the patient survival time, the survival curves were used for visualization of the model. The specific risk scores value of each sample in the model was visualized with $\mathrm{R}$ software. The irlncRNAs in the risk model were used to perform PCA on all samples, and the accuracy of the risk model was evaluated by comparing the PCA results of 224 irLncRNA, immune genes, and whole gene expression profiles.

\section{Correlation analysis of risk model with clinicopathological features}

The relationship between the model and clinicopathological features of CSCC patients was evaluated with a Chi-square test. These features included age, grade, clinical stage, TNM stage. Tumor mutational burden (TMB) and microsatellite instability (MSI) were also 
be analyzed. The risk scores difference between the groups corresponding to these clinicopathological characteristics was calculated with the Wilcoxon rank-sum test.

\section{Pathways in two risk groups}

To figure out the significantly changed immunological pathways, we did a GSEA[42] for two risk groups. We selected significantly enriched pathways according to $p$-value and FDR q-value, they were less than 0.05 and 0.25 , respectively.

\section{Immune cell infiltration analysis}

For the connection between risk and immune cell characteristics, we calculated the amount of immune cell infiltration in TCGA cervical cancer samples. The immune cell data were mainly obtained from XCELL, TIMER, QUANTISEQ, MCPCOUNTER, EPIC, CIBERSORT, and CIBERSORT-ABS. We used Spearman correlation analysis to evaluate the association between the risk scores and immune infiltrating cells. According to the immune cells infiltration data in CIBERSORT, we assessed the interaction among these immune cells and also the correlation between 11 irlncRNA and immune cells.

\section{Exploring the immunotherapeutic treatment of the risk model}

The Wilcoxon's test was used to explore the differential expression of ICP( PD-L1, PD-1, CTLA-4, TIGIT, LAG- 3, and TIM-3) between two risk groups. Then we used the TIDE algorithm to predict the possibility of immunotherapy response, which is a computational framework expanded for immunotherapy forecast, and its predictive function has been successfully verified[43].

\section{Non-negative Matrix Factorization}

To assess if any clusters were present in the CSCC cohorts, molecular subtyping was used to perform NMF. The best $r$ value was chosen according to quality factors. These factors were the first r-value at which the concord coefficient began to decline and the first value at which the residual sum of squares curve appeared to have an inflection point, as well as a direct visual inspection of the consensus matrix. The association of two subtypes and risk groups was showed in Sankey Diagram. We also explored the expression of immune cells and immune checkpoints proteins in two subtypes. PCA was performed with the R package ggplot2.

\section{Exploration of drug therapy targeting risk model}

To determine if the models we constructed could be used for drug therapy in CSCC patients, we calculated the IC50 of common chemotherapy drugs in the CSCC dataset. Anti-tumor drugs such as cisplatin, paclitaxel, docetaxel, gemcitabine, and vinorelbine were advocated for the treatment of CSCC patients. The IC50 difference between the two risk groups was compared by Wilcoxon's test. Results were plotted with R packages "pRRophetic" and "ggplot2".

\section{Establishing a predictive nomogram}

A nomogram combined various factors, and a calibration curve evaluated whether the predicted survival was consistent with certain survival in the nomogram. Calibration curves and AUC-ROC were also used to appraise the predictive ability of the prognostic model, and the clinical efficacy of the nomogram was evaluated with the DCA.

\section{Declarations}

\section{Acknowledgements}

We thank the researchers who gave their data for this analysis. And we also would like to acknowledge the GTEx and TCGA database.

\section{Authors' contributions}

X.L conceived the manuscript , X.L, L.L and Y.Y participated in data collection and processing, X.L , L.L and P.L analyzed and interpreted the data, X.L, M.P and H.J participated in manuscript writing. All authors read and approved the final manuscript.

\section{Additional information \\ Competing interests}

The authors declare that they have no competing interests. 


\section{Availability of data and materials}

The datasets supporting the conclusions of this article are available in the Genotype-Tissue Expression (GTEx) database and the cancer genome atlas (TCGA) database.

\section{Funding}

This study was funded by grants from the Scientific and Technological Project of Henan Province (172102310077). Funds for Creative Research Team of Henan Province.

\section{References}

1. Sawaya, G. F., Smith-McCune, K. \& Kuppermann, M. Cervical Cancer Screening: More Choices in 2019., 321 (20), 2018-2019 (2019).

2. Wen, X., Liu, S. \& Cui, M. Effect of BRCA1 on the Concurrent Chemoradiotherapy Resistance of Cervical Squamous Cell Carcinoma Based on Transcriptome Sequencing Analysis. Biomed Res Int, 2020, 3598417 (2020).

3. Kumar, L., Harish, P., Malik, P. S. \& Khurana, S. Chemotherapy and targeted therapy in the management of cervical cancer. Curr Probl Cancer, 42 (2), 120-128 (2018).

4. Sol, E. S. et al. Comparison of concurrent chemoradiotherapy with cisplatin plus 5-fluorouracil versus cisplatin plus paclitaxel in patients with locally advanced cervical carcinoma. J Gynecol Oncol, 20 (1), 28-34 (2009).

5. Marquina, G., Manzano, A. \& Casado, A. Targeted Agents in Cervical Cancer: Beyond Bevacizumab. Curr Oncol Rep, 20 (5), 40 (2018).

6. Long, J. et al. PD-1/PD-L blockade in gastrointestinal cancers: lessons learned and the road toward precision immunotherapy. $J$ Hematol Oncol, 10 (1), 146 (2017).

7. Chung, H. C. et al. Efficacy and Safety of Pembrolizumab in Previously Treated Advanced Cervical Cancer: Results From the Phase II KEYNOTE-158 Study. J Clin Oncol, 37 (17), 1470-1478 (2019).

8. Kung, J. T., Colognori, D. \& Lee, J. T. Long noncoding RNAs: past, present, and future., 193 (3), 651-669 (2013).

9. Statello, L., Guo, C. J., Chen, L. L. \& Huarte, M. Gene regulation by long non-coding RNAs and its biological functions. Nat Rev Mol Cell Biol, 22 (2), 96-118 (2021).

10. Denaro, N., Merlano, M. C. \& Lo Nigro, C. Long noncoding RNAs as regulators of cancer immunity. Mol Oncol, 13 (1), 61-73 (2019).

11. Zhang, L., Xu, X. \& Su, X. Noncoding RNAs in cancer immunity: functions, regulatory mechanisms, and clinical application. Mol Cancer, 19 (1), 48 (2020).

12. Sang, L. J. et al. LncRNA CamK-A Regulates $\mathrm{Ca}(2+)$-Signaling-Mediated Tumor Microenvironment Remodeling. Mol Cell, 72 (1), 71-8377 (2018).

13. Zhang, J. et al. LncRNA HLA-F-AS1 promotes colorectal cancer metastasis by inducing PFN1 in colorectal cancer-derived extracellular vesicles and mediating macrophage polarization. Cancer Gene Ther2021.

14. Jiang, R. et al. The long noncoding RNA Inc-EGFR stimulates T-regulatory cells differentiation thus promoting hepatocellular carcinoma immune evasion. Nat Commun, 8, 15129 (2017).

15. Zhang, Y., Liu, Q. \& Liao, Q. Long noncoding RNA: a dazzling dancer in tumor immune microenvironment. J Exp Clin Cancer Res, 39 (1), 231 (2020).

16. Wang, W. et al. An immune-related IncRNA signature for patients with anaplastic gliomas. J Neurooncol, 136 (2), 263-271 (2018).

17. Li, Y. et al. Pan-cancer characterization of immune-related IncRNAs identifies potential oncogenic biomarkers. Nat Commun, 11 (1), 1000 (2020).

18. Zhang, Y. Y., Li, M., Xu, Y. D. \& Shang, J. LncRNA SNHG14 promotes the development of cervical cancer and predicts poor prognosis. Eur Rev Med Pharmacol Sci, 23 (9), 3664-3671 (2019).

19. Hao, Y. et al. IL-6/STAT3 mediates the HPV18 E6/E7 stimulated upregulation of MALAT1 gene in cervical cancer HeLa cells. Virus Res, 281, 197907 (2020). 
20. Shao, S., Wang, C., Wang, S., Zhang, H. \& Zhang, Y. LncRNA STXBP5-AS1 suppressed cervical cancer progression via targeting miR-96-5p/PTEN axis. Biomed Pharmacother, 117, 109082 (2019).

21. Liu, C., Tian, X., Zhang, J. \& Jiang, L. Long Non-coding RNA DLEU1 Promotes Proliferation and Invasion by Interacting With miR381 and Enhancing HOXA13 Expression in Cervical Cancer. Front Genet, 9, 629 (2018).

22. Gasser, S., Lim, L. H. K. \& Cheung, F. S. G. The role of the tumour microenvironment in immunotherapy. Endocr Relat Cancer, 24 (12), T283-t295 (2017).

23. Chen, Q., Li, T. \& Yue, W. Drug response to PD-1/PD-L1 blockade: based on biomarkers. Onco Targets Ther, 11, 4673-4683 (2018).

24. Garon, E. B. et al. Five-Year Overall Survival for Patients With Advanced Non-Small-Cell Lung Cancer Treated With Pembrolizumab: Results From the Phase I KEYNOTE-001 Study. J Clin Oncol, 37 (28), 2518-2527 (2019).

25. Qian, B. Z. \& Pollard, J. W. Macrophage diversity enhances tumor progression and metastasis., 141 (1), $39-51$ (2010).

26. Aran, D., Hu, Z. \& Butte, A. J. xCell: digitally portraying the tissue cellular heterogeneity landscape. Genome Biol, 18 (1), 220 (2017).

27. Plattner, C., Finotello, F. \& Rieder, D. Deconvoluting tumor-infiltrating immune cells from RNA-seq data using quanTIsEq. Methods Enzymol, 636, 261-285 (2020).

28. Dienstmann, R. et al. Relative contribution of clinicopathological variables, genomic markers, transcriptomic subtyping and microenvironment features for outcome prediction in stage II/III colorectal cancer. Ann Oncol, 30 (10), 1622-1629 (2019).

29. Racle, J., de Jonge, K., Baumgaertner, P., Speiser, D. E. \& Gfeller, D. Simultaneous enumeration of cancer and immune cell types from bulk tumor gene expression data. Elife2017, 6 .

30. Li, T. et al. TIMER: A Web Server for Comprehensive Analysis of Tumor-Infiltrating Immune Cells. Cancer Res, 77 (21), e108-e110 (2017).

31. Li, T. et al. TIMER2.0 for analysis of tumor-infiltrating immune cells. Nucleic Acids Res, 48 (W1), W509-w514 (2020).

32. Zhang, H. et al. Poor Clinical Outcomes and Immunoevasive Contexture in Intratumoral IL-10-Producing Macrophages Enriched Gastric Cancer Patients.Ann Surg2020.

33. Tamminga, M. et al. Immune microenvironment composition in non-small cell lung cancer and its association with survival. Clin Transl Immunology, 9 (6), e1142 (2020).

34. Finn, O. J. Immuno-oncology: understanding the function and dysfunction of the immune system in cancer. Ann Oncol, 23 (Suppl 8(Suppl 8), viii6-9 (2012).

35. Eskander, R. N. \& Tewari, K. S. Immunotherapy: an evolving paradigm in the treatment of advanced cervical cancer. Clin Ther, 37 (1), 20-38 (2015).

36. Enwere, E. K. et al. Expression of PD-L1 and presence of CD8-positive T cells in pre-treatment specimens of locally advanced cervical cancer. Mod Pathol, 30 (4), 577-586 (2017).

37. Dyer, B. A., Zamarin, D., Eskandar, R. N. \& Mayadev, J. M. Role of Immunotherapy in the Management of Locally Advanced and Recurrent/Metastatic Cervical Cancer. J Natl Compr Canc Netw, 17 (1), 91-97 (2019).

38. Heeren, A. M. et al. Prognostic effect of different PD-L1 expression patterns in squamous cell carcinoma and adenocarcinoma of the cervix. Mod Pathol, 29 (7), 753-763 (2016).

39. Karim, R., Jordanova, E. \& Piersma, S. Tumor-expressed B7-H1 and B7-DC in relation to PD-1 + T-cell infiltration and survival of patients with cervical carcinoma. Clin Cancer Res, 15, 6341-6347 (2009).

40. Lorusso, D., Petrelli, F., Coinu, A., Raspagliesi, F. \& Barni, S. A systematic review comparing cisplatin and carboplatin plus paclitaxel-based chemotherapy for recurrent or metastatic cervical cancer. Gynecol Oncol, 133 (1), 117-123 (2014).

41. Sauerbrei, W., Royston, P. \& Binder, H. Selection of important variables and determination of functional form for continuous predictors in multivariable model building. Stat Med, 26 (30), 5512-5528 (2007).

42. Mootha, V. K. et al. PGC-1alpha-responsive genes involved in oxidative phosphorylation are coordinately downregulated in human diabetes. Nat Genet, 34 (3), 267-273 (2003).

43. Xu, F. et al. A signature of immune-related gene pairs predicts oncologic outcomes and response to immunotherapy in lung adenocarcinoma., 112 (6), 4675-4683 (2020). 
Figures

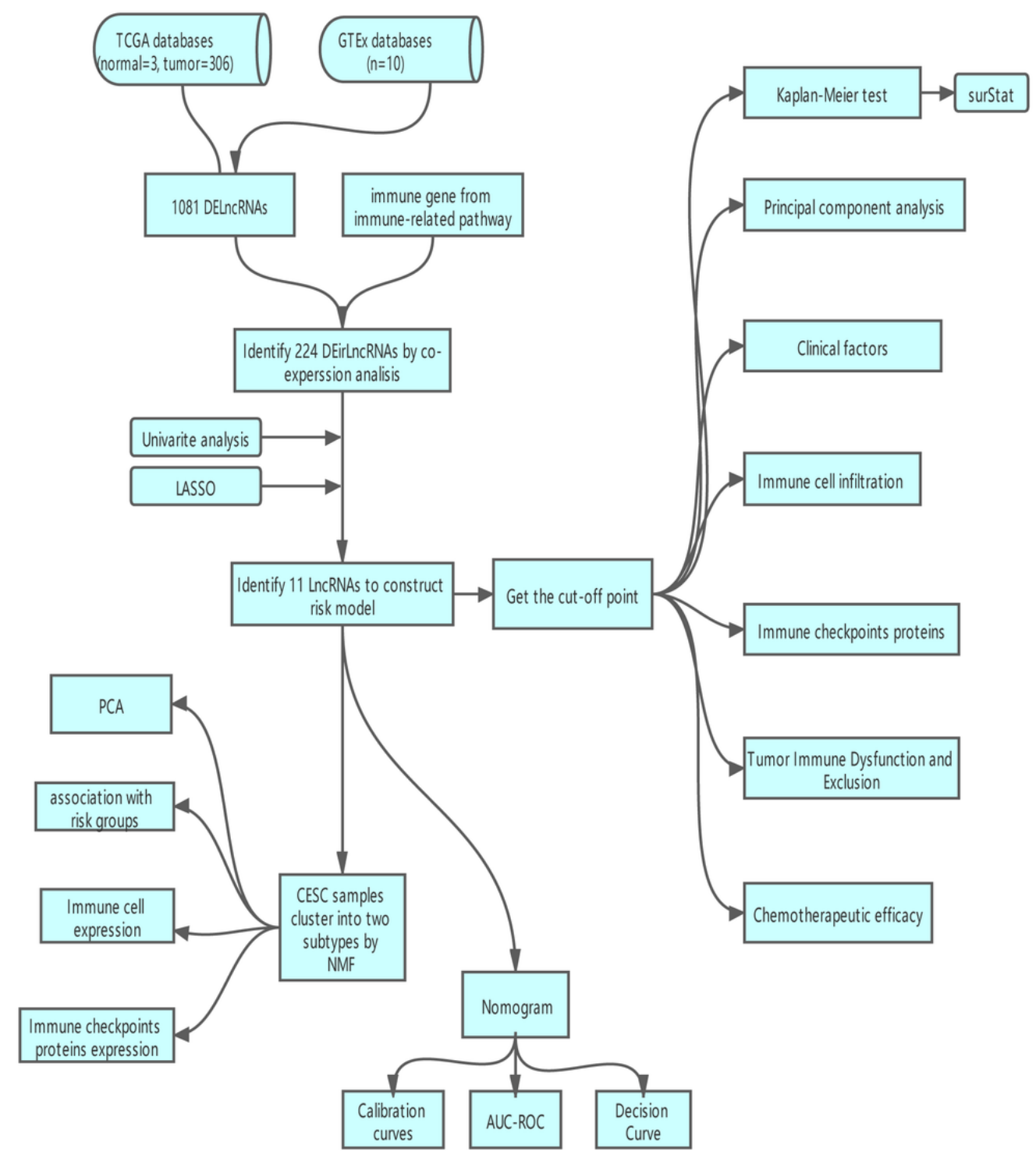

Figure 1

Flow chart of this study 


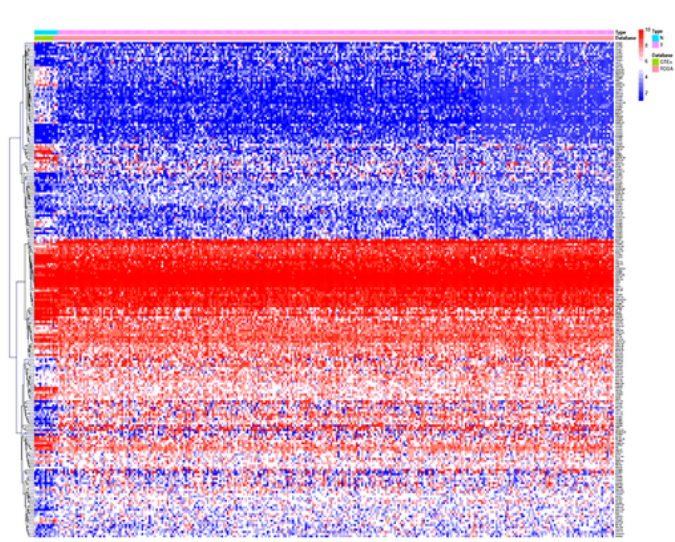

C

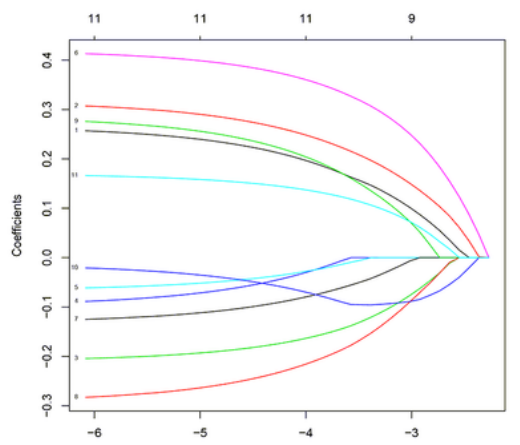

d

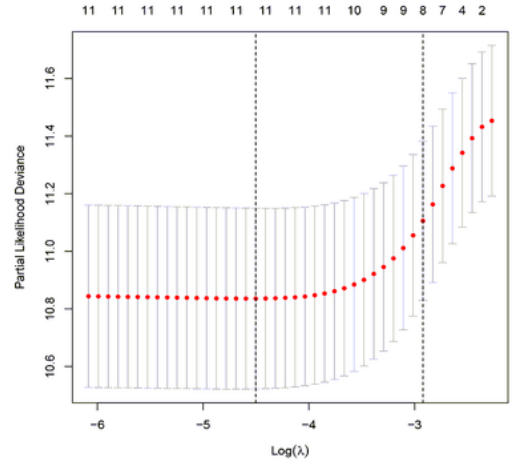

b
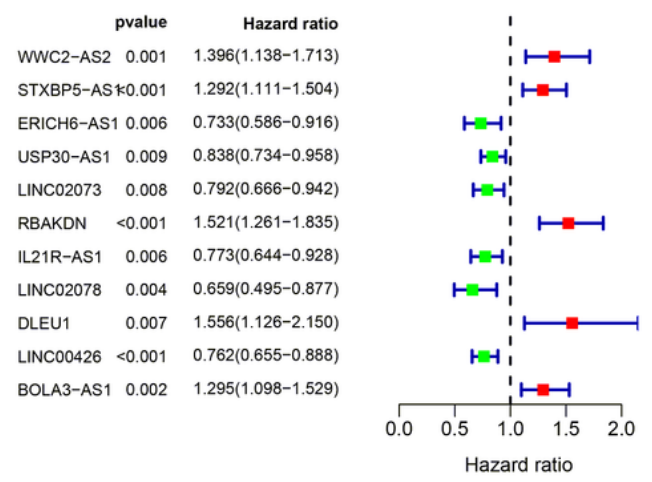

e

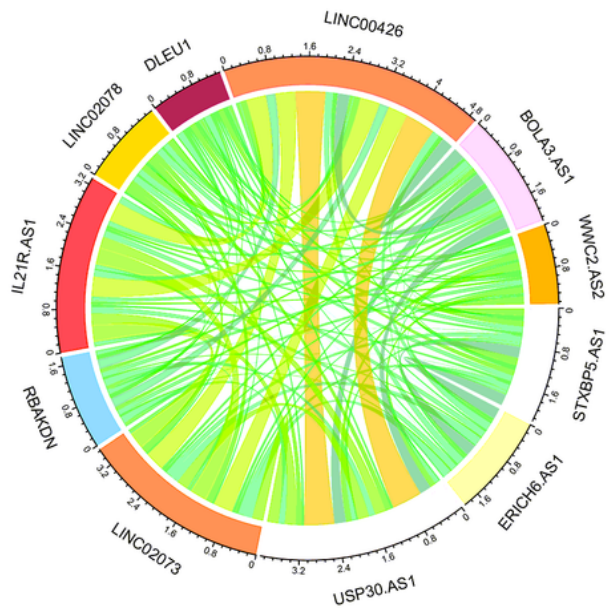

\section{Figure 2}

Screen immune-related IncRNA to construct a model. (a) Heatmap of 224 DEirLncRNA (b) forest map of univariate Cox analysis (c) Distribution of the LASSO coefficients for 11 IncRNAs (d) partial likelihood deviation of the LASSO coefficient distribution. Vertical dashed lines indicate lambda; e correlation chord diagram between 11 IncRNAs 
a
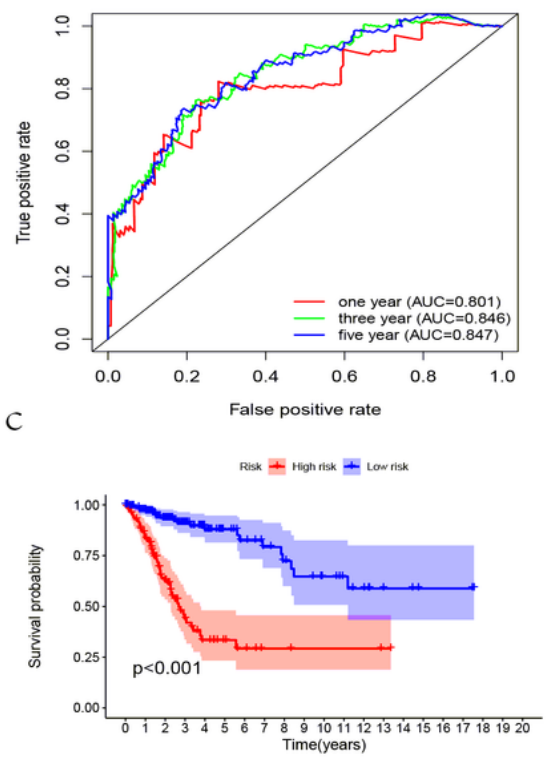

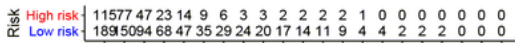
18950946847352924201714119444222
$01 \dot{2} 345678$
Time(years)

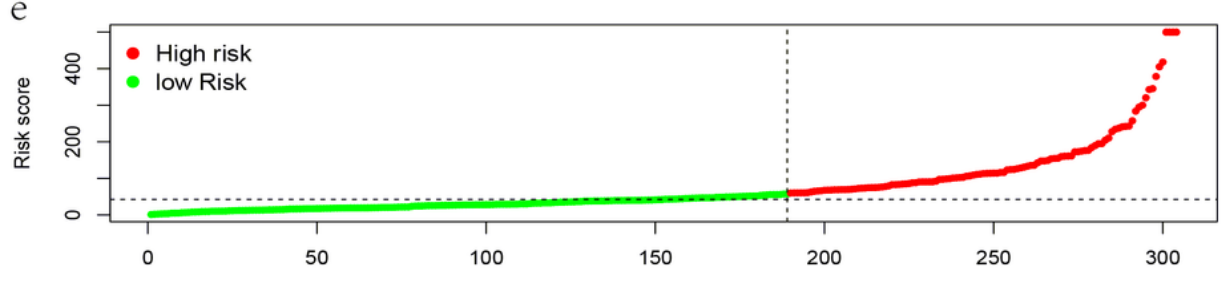

f Patients (increasing risk socre)

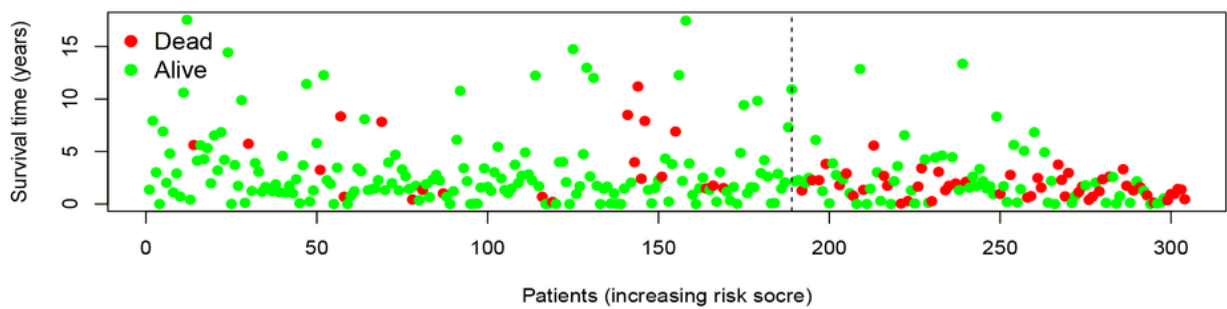

\section{Figure 3}

Validation of the risk model. (a) The 1-, 3-, and 5-year ROC of the risk model (b) the maximum inflection point is the cut-off point obtained by the AIC (c) Patients in the low-risk group experienced a longer survival time tested by the Kaplan-Meier test (d-f) risk scores distribution, survival status and survival time and expression profile of 11 IncRNAs in the CESC patients 
a
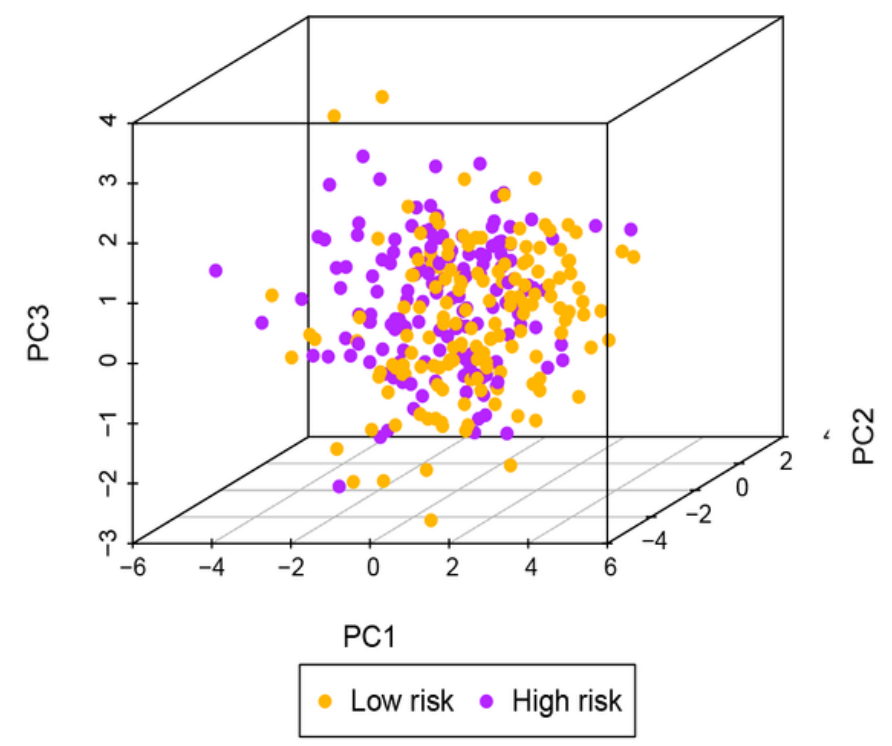

C

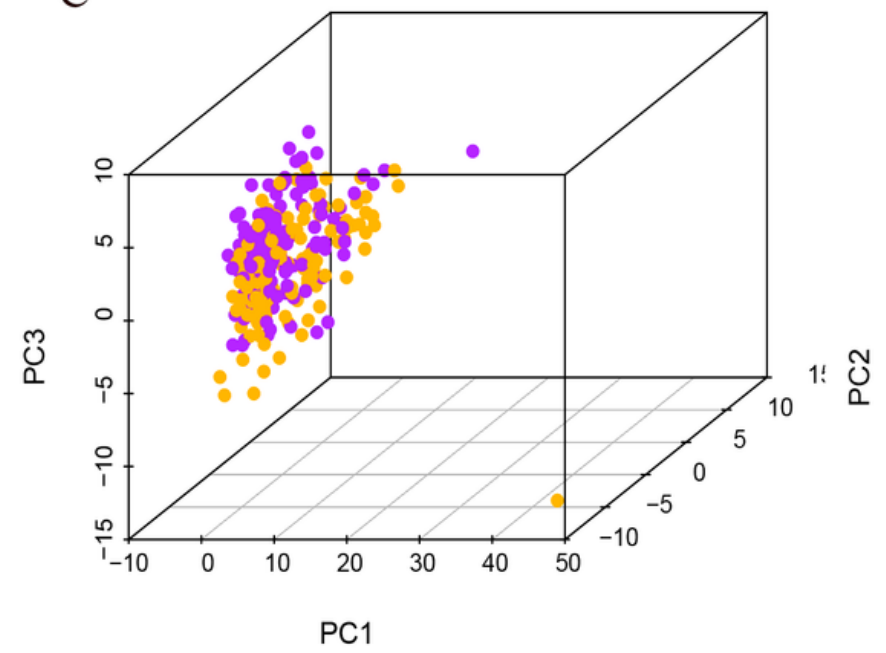

b
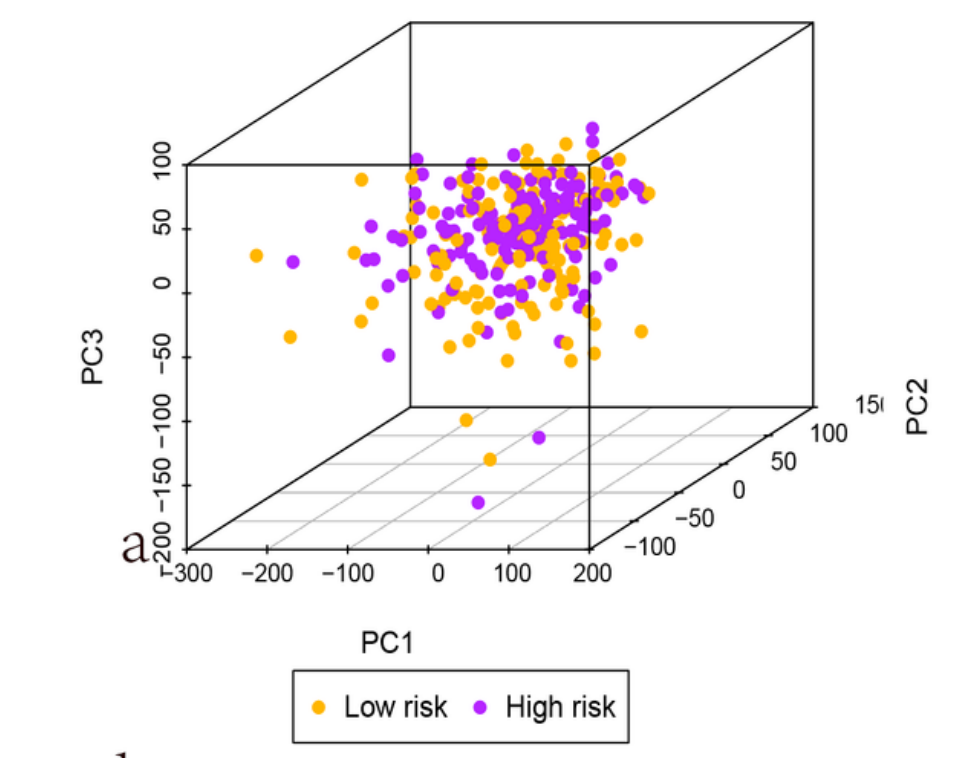

d

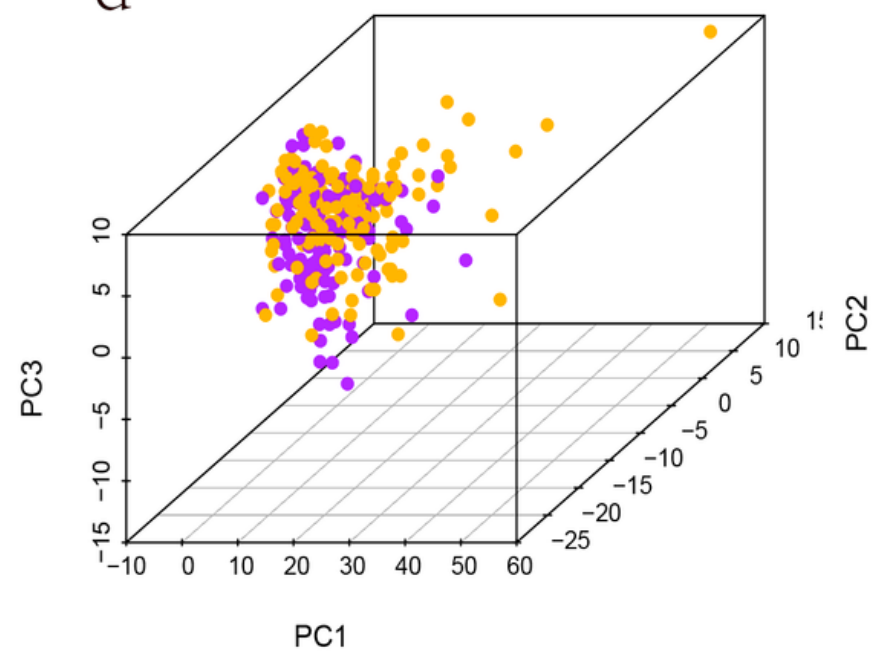

Figure 4

Principal component analysis between the high- and low-risk groups based on (a) IncRNAsin risk model (b) 224 DEirlncRNA (c) immune genes (d) and whole gene expression profiles. 
a

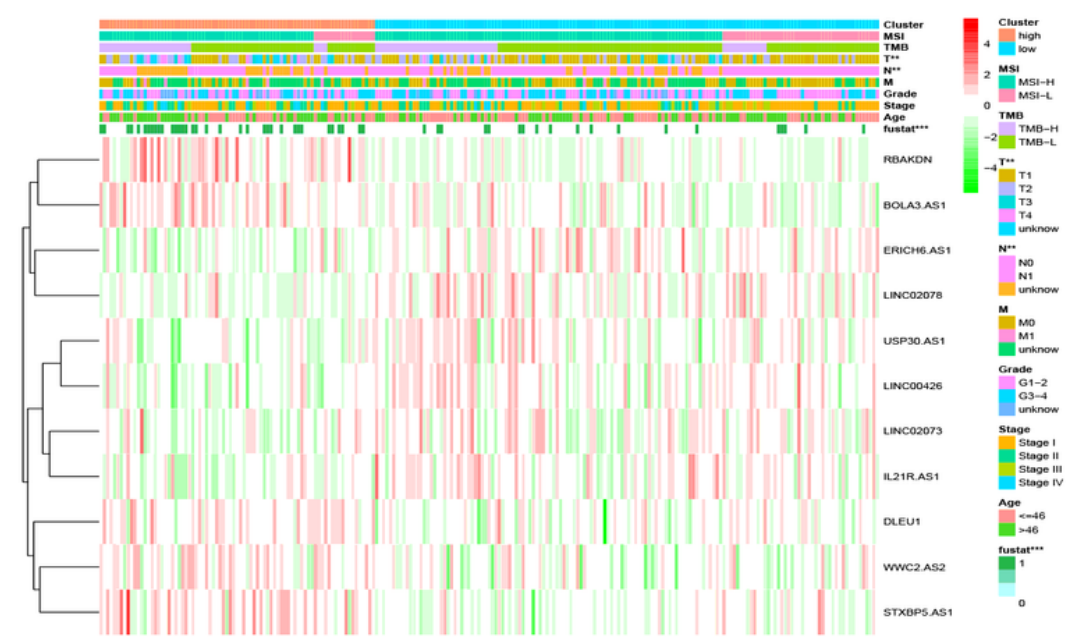

b
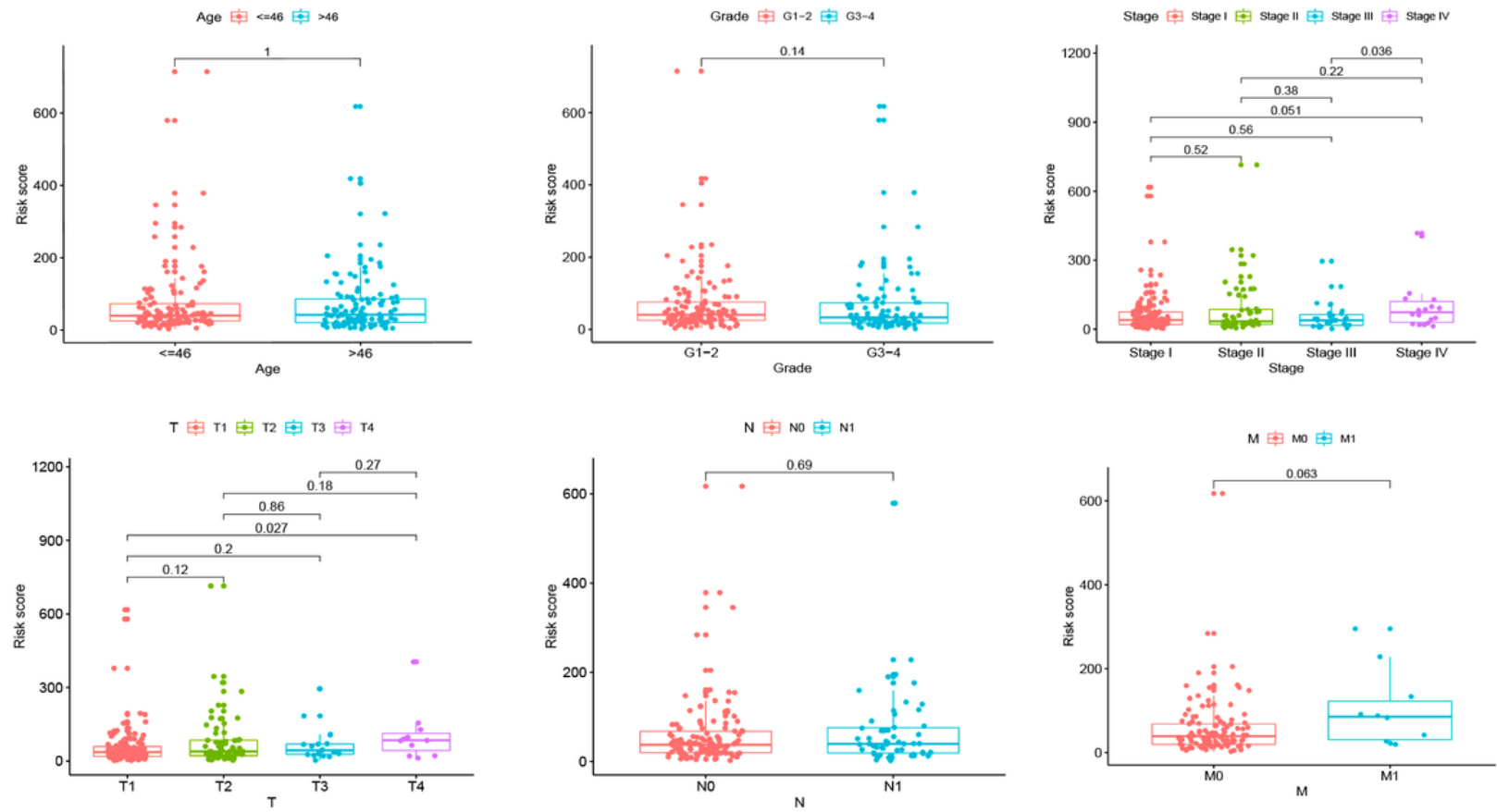

\section{Figure 5}

Correlation analyses of risk scores with clinicopathological characteristics of CESC patients. (a) Heatmap and clinicopathologic features of high- and low -risk groups $(<0.001=* \star *,<0.01=* \star *$, and $<0.05=*)$. Chi-square test acted as the statistical significance test distribution of risk scores in N classification. age, grade, clinical stage, and TNM stage. (b) Wilcoxon rank-sum or Kruskal-Wallis rank-sum test acted as the statistical significance test. The clinical stage and T stage were significantly associated with the risk scores. 

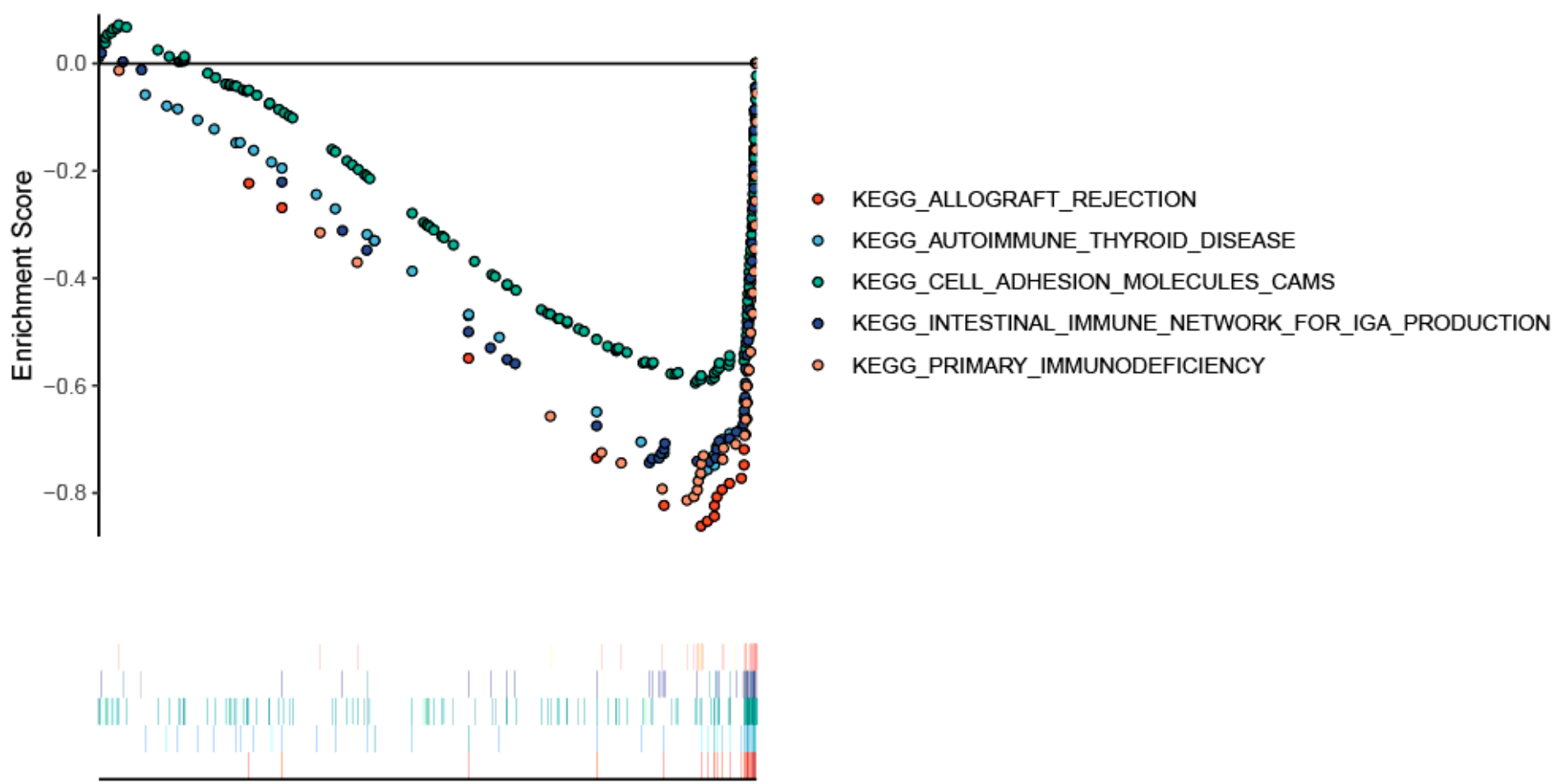

High risk<-------------> Low risk

Figure 6

Gene set enrichment analysis. 
a

c
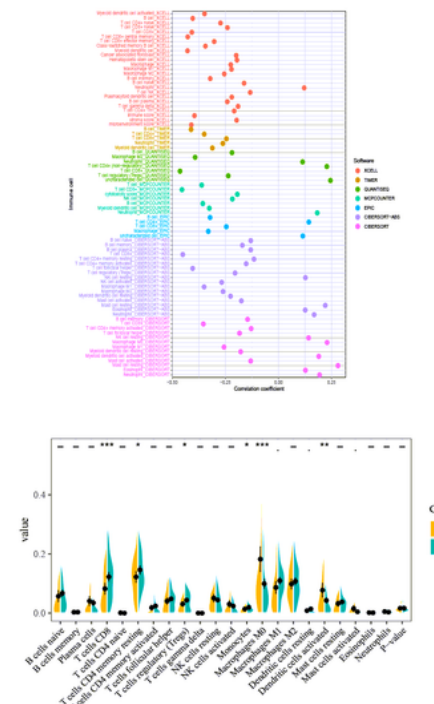

d

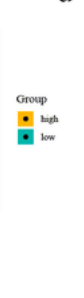

b
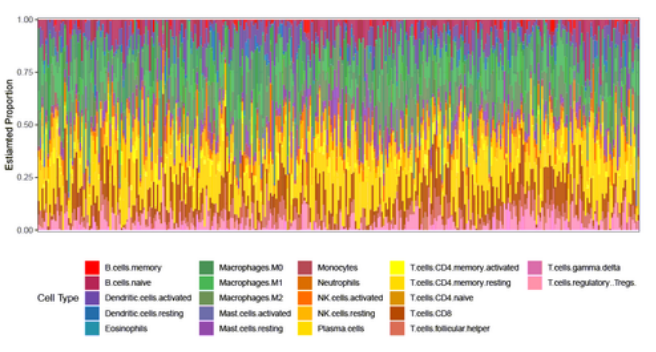

e

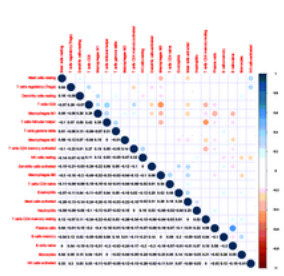

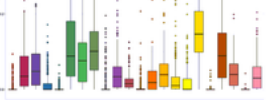

f

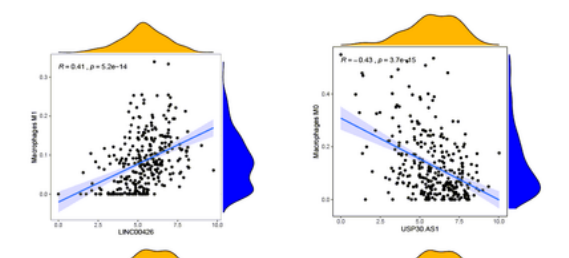

-
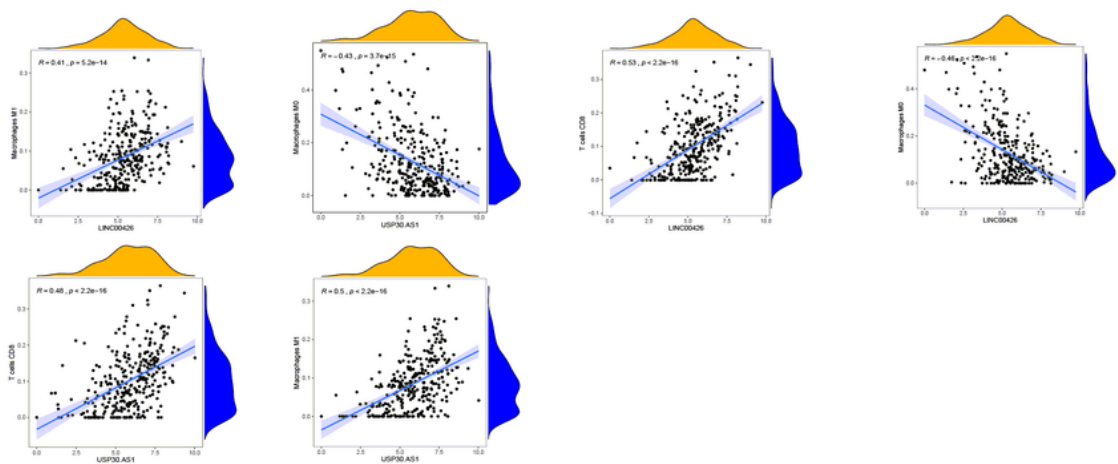

g

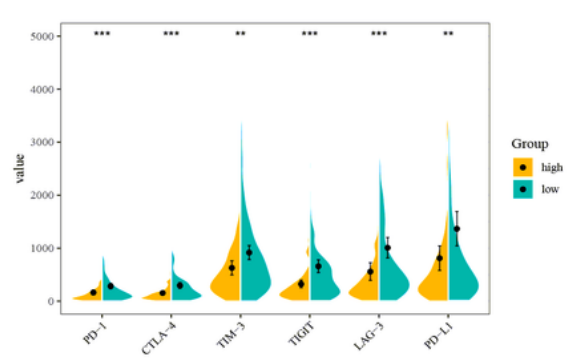

h

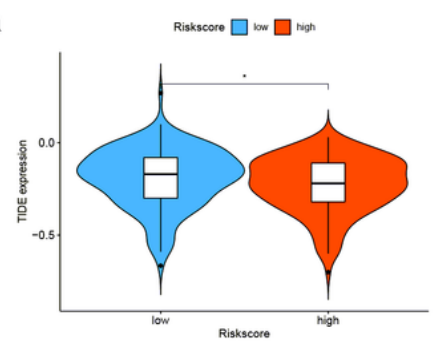

Figure 7

Immune infiltrate landscape in CESC patients and estimation of immunosuppressed molecules by the risk model. (a) Correlation between immune cells and risk scores (b) relative proportion of immune cell infiltrates in CESC patients (c) comparison of the expression of immune infiltrating cells in the low- and high-risk patients (d) Content of 22 immune cells (e) Correlation matrix of immune cells (f) Correlation of irlncRNAs and immune cells (g) Differential expression of immune checker proteins in the high- and low-risk patients (h) TIDE prediction difference in the high- and low-risk patients 

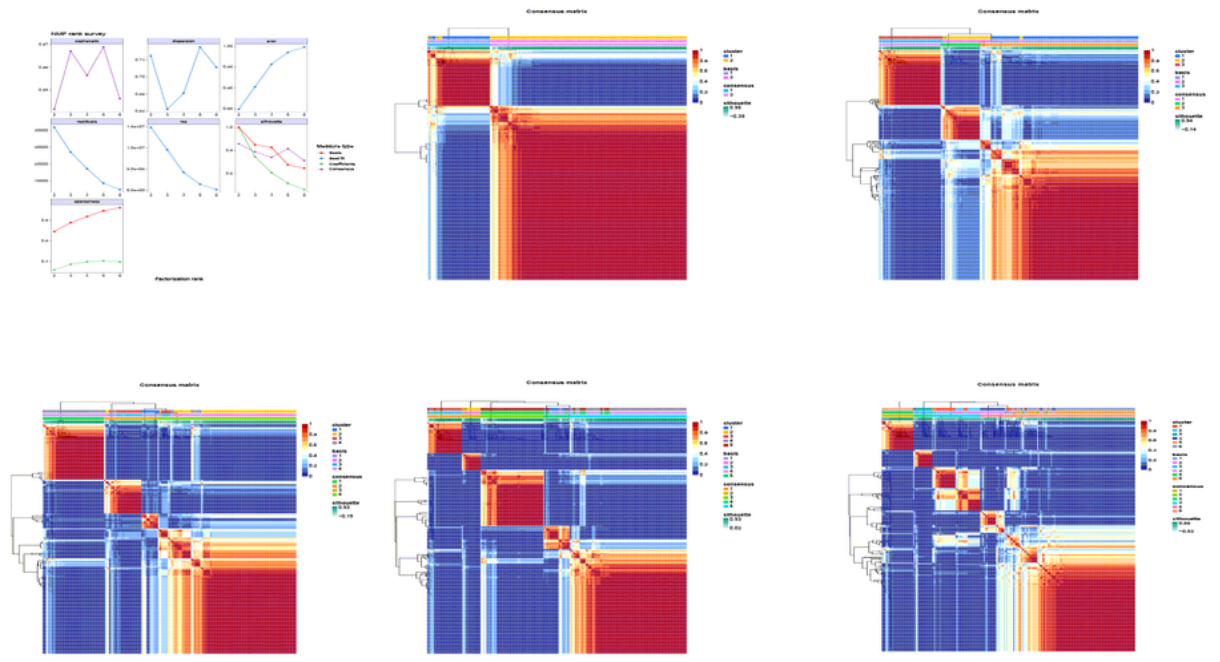

b

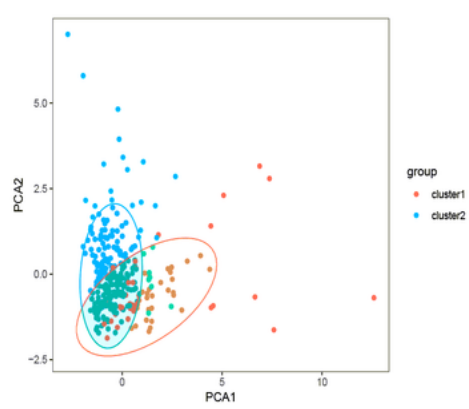

d

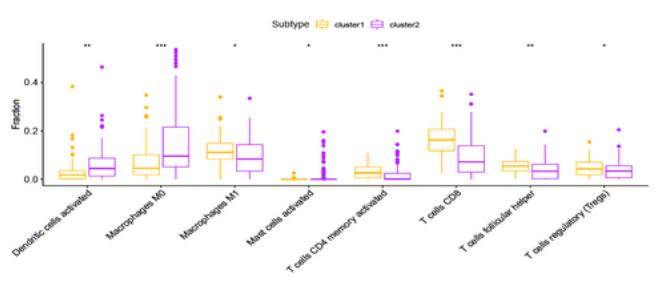

C

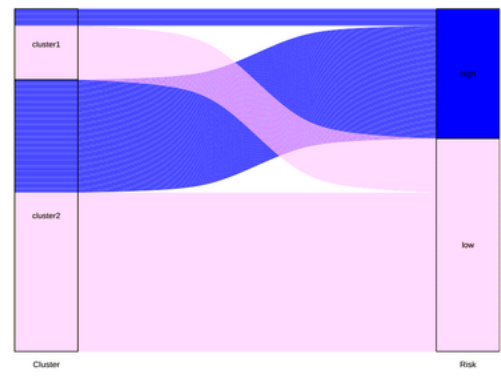

e

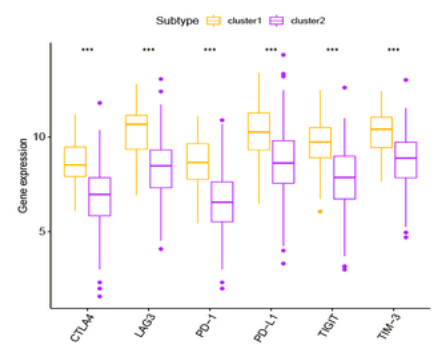

\section{Figure 8}

CESC patients clustered into two subtypes based on risk mode. (a) consensus clustering of CESC samples by NMF. correlation matrix heatmaps correspond to $r$ ranks 2-6 (b) PCA plots of CESC samples (c) association of subtypes and risk groups (d) PD-L1, PD-1, CTLA-4, TIGIT, LAG- 3, and TIM-3 in cluster 1 had significantly higher expression than in cluster 2 (e) differences in the expression of immune cells between the two subtypes 

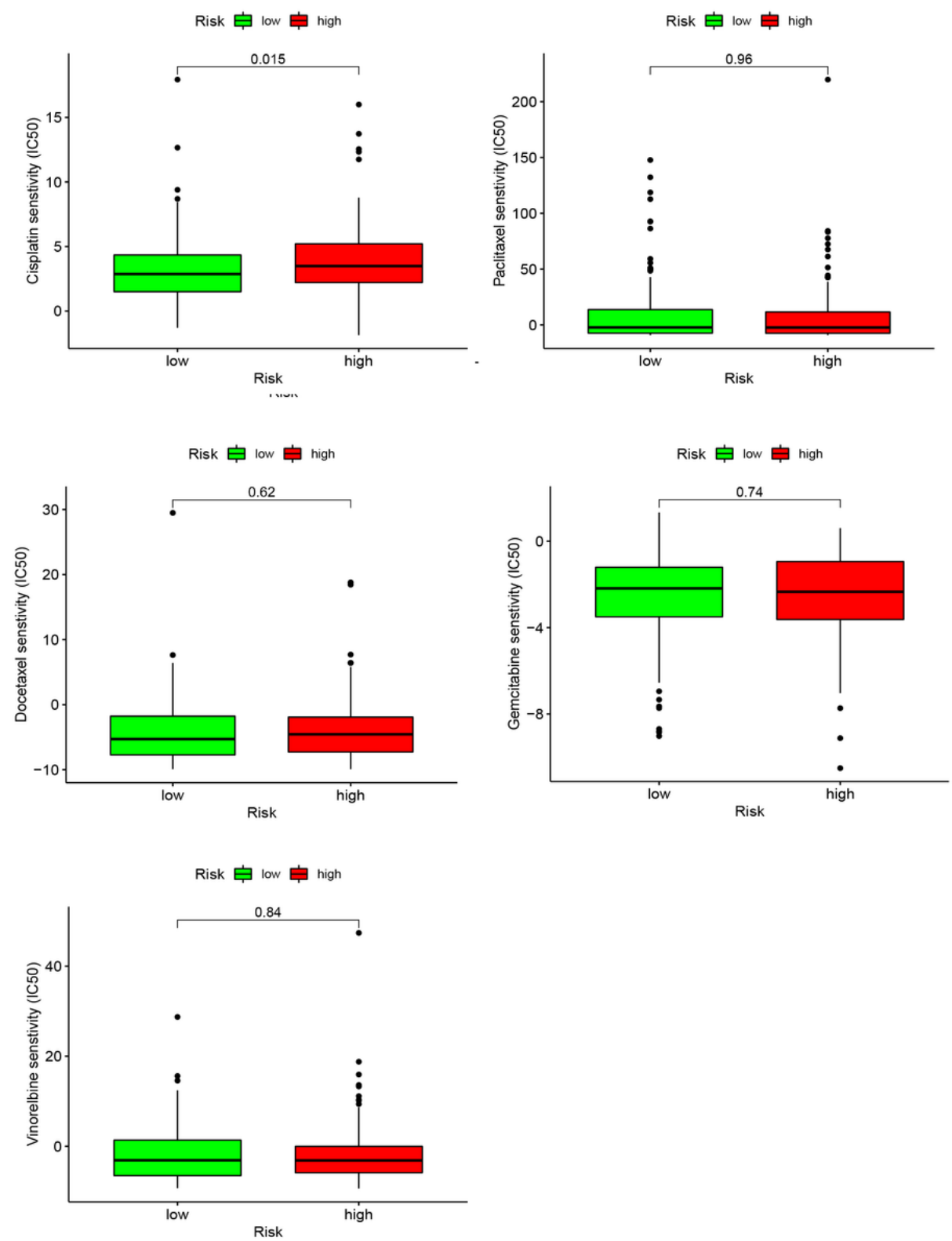

Figure 9

Sensitivity of chemotherapy drugs in high- and low-risk groups. 
a
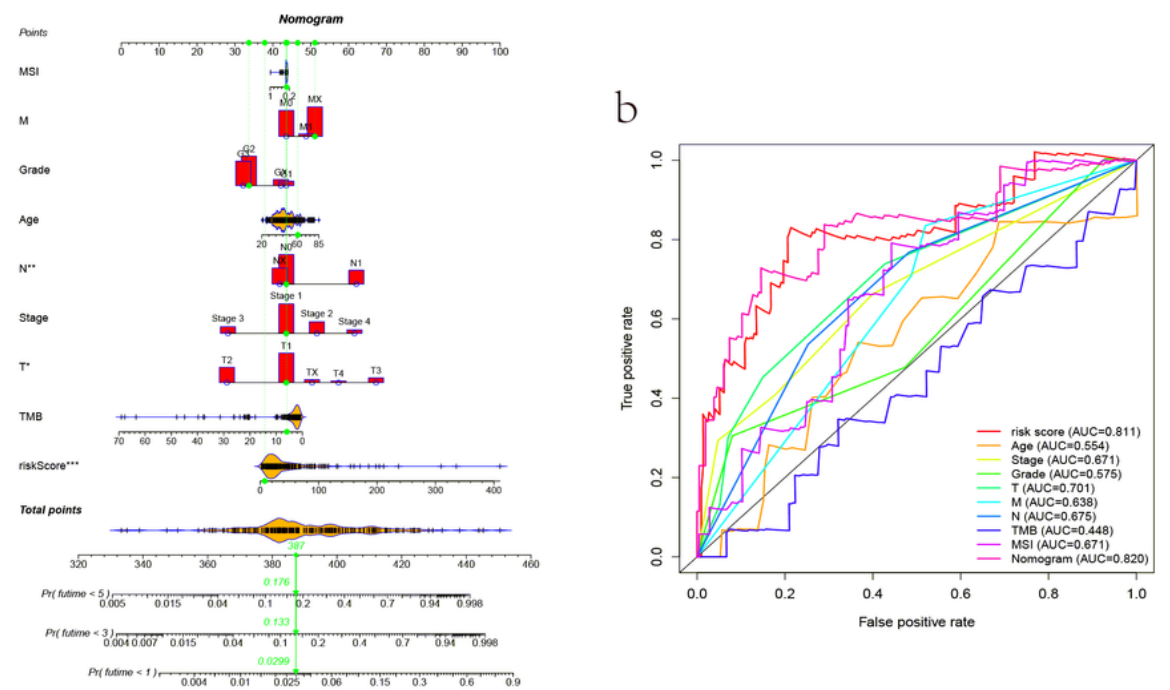

C
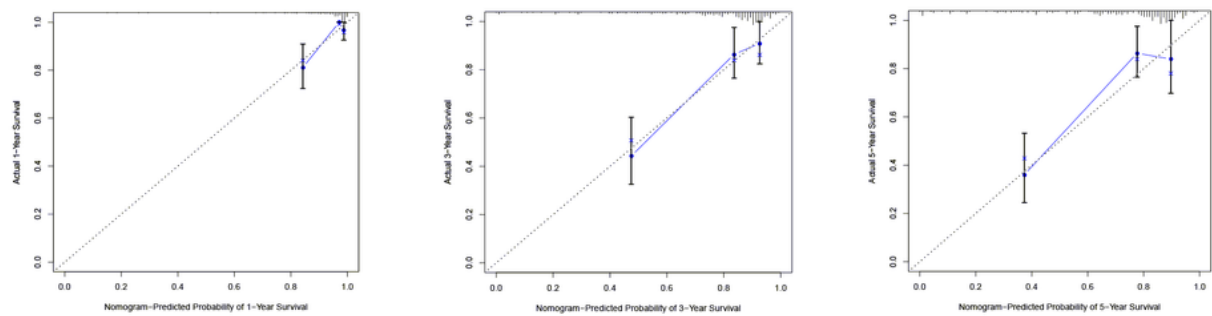

d

e
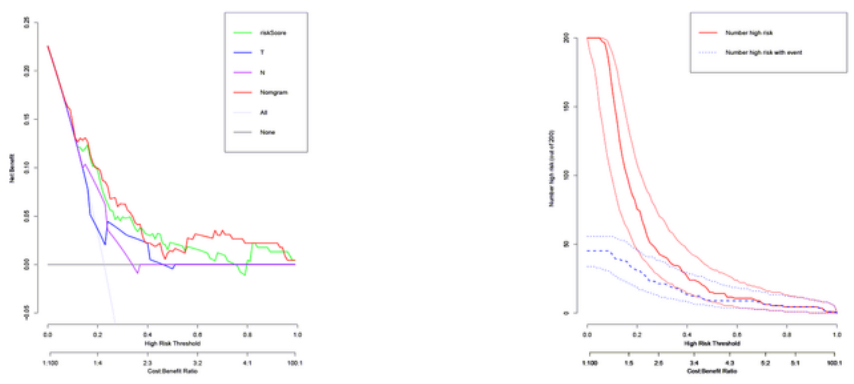

\section{Figure 10}

Construction and evaluation of a prognostic nomogram. (a) The nomogram predicts the probability of the 1-, 3-, and 5-year OS (b) the AUC of risk scores and clinical factors (c) the calibration plot of the nomogram predicts the probability of the 1-, 3-, and 5-year OS. (d) the decision curve of nomogram; (e) clinical impact curve 